\title{
La comprensión lectora del idioma inglés mediante actividades lúdicas
}

\section{Reading comprehension of English language through playful activities}

Luis Even Pazmiño Pavón. ${ }^{1}$, Paúl Rolando Armas Pesántez. ${ }^{2}$, Edison Hernán Salazar Calderón. ${ }^{3}$ \& Edgar Eduardo Heredia Arboleda. ${ }^{4}$

\section{Recibido: 12-12-2019 / Revisado: 01-12-2019 / Aceptado: 06-01-2020 / Publicado: 07-02-2020}

Resumen

DOI: https://doi.org/10.33262/concienciadigital.v3i1.1.1134

El objetivo de la presente investigación, fue realizar un diagnóstico de la comprensión lectora del idioma inglés mediante la aplicación de actividades lúdicas tales como: cuentos, juegos y vocabulario, en estudiantes del segundo nivel de Inglés de la Carrera de Agroindustria de la Facultad de Ciencias Pecuarias de la Escuela Superior Politécnica de Chimborazo durante el periodo académico septiembre 2019 - febrero 2020. Ya que se conoce que los estudiantes experimentan falta de concentración. La investigación tuvo un enfoque educativo, de tipo descriptiva, bibliográfica de campo, cuali cuantitativa y temporal. Para alcanzar la meta se elaboró una ficha de observación, la misma que fue revisada y aprobado por un grupo de cuatro pares académicos especialistas del área de inglés. La misma que utilizó un rango de opciones según una escala de Likert, que permitió medir aspectos cualitativos para posteriormente cuantificarlos. La muestra fue intencional, compuesta por 30 estudiantes entre hombres y mujeres, que tienen similares conocimientos del idioma inglés. La información obtenida fue tabulada y representada en tablas y gráficos estadísticos para posteriormente analizarla e interpretarla, permitiendo determinar que la aplicación de las actividades lúdicas son estrategias que motivan la comprensión lectora del idioma inglés. Se recomienda integrar

1 Escuela Superior Politécnica de Chimborazo, Centro de Idiomas, Riobamba, Ecuador, luis.pazminio@espoch.edu.ec

2 Escuela Superior Politécnica de Chimborazo, Facultad de Ciencias, Riobamba, Ecuador, parmas@espoch.edu.ec

3 Escuela Superior Politécnica de Chimborazo, Facultad de Ciencias Pecuarias, Riobamba, Ecuador, edisonh.salazar@espoch.edu.ec

4 Universidad Nacional de Chimborazo, Facultad de Ciencias de la Educación, Humana y Tecnología, Riobamba, Ecuador, edu1987h@hotmail.com 
las actividades lúdicas como estrategia en el plan de estudios para incidir en la comprensión lectora de inglés.

Palabras claves: Comprensión lectora, Actividades lúdicas, cuento, juego, vocabulario, inglés.

\section{Abstract}

The objective of the present research work was to make a diagnosis of the reading comprehension of English language by means of applying playful activities such as stories, games, and vocabulary on students of second English Level of Agroindustry Engineering at the Animal Science Faculty of the Escuela Superior Polytechnical de Chimborazo during the academic period September 2019 - February 2020. Since it is known that students experience a lack of concentration, the research had an educational, descriptive, bibliographic, qualitative, quantitative and temporal approach. To achieve the objective, an observation form was prepared, which was reviewed and approved by a group of four academic peers who are specialized in English. The form used a range of options according to a Likert scale, which allowed to measure qualitative aspects to later quantify them. The sample was intentional, composed of 30 students, men and women, who have similar knowledge of the English language. The information obtained was tabulated and represented in statistical tables and graphs to later be analyzed and interpreted, allowing to determine that the application of playful activities are strategies that motivate the reading comprehension of the English language. It is recommended to integrate recreational activities as a strategy in the curriculum to influence reading comprehension of English.

Keywords: Reading comprehension, Playful activities, story, game, vocabulary, English

\section{Introducción}

El conocimiento del idioma inglés, es una necesidad básica para docentes y estudiantes que nos ayuda a tener ventajas competitivas en el ambiente laboral y en nuestra vida diaria. No es fácil dominar el idioma cuando el estudiante es adulto, siempre pone resistencia debido a que no es su lenguaje nativo y su desarrollo mental ya se encuentra en etapas posteriores a las de un niño, estos factores son uno de los tantos que se evidencia cuando estamos en el aula, por lo tanto, es prioridad encontrar metodologías didácticas que motiven aprender el idioma, y que den resultados en los aprendizajes significativos. 
ISSN: 2600-5859

Según Díaz (2002), indica que se conoce como lúdico, al adjetivo que designa todo aquello relativo al juego, ocio, entretenimiento o diversión. El término lúdico es de origen latín "ludus" que significa "juego"

Kintsch (1998) indica que una actividad lúdica es realizada en el tiempo libre de los individuos, con el objetivo de liberar tensiones, huir de la rutina diaria y preocupaciones, para obtener un poco de placer, diversión y entretenimiento, así como otros beneficios, entre los cuales están:

- $\quad$ Amplia la expresión corporal.

- $\quad$ Desenvuelve la concentración y agilidad mental.

- $\quad$ Mejora el equilibrio y flexibilidad.

- Aumenta la circulación sanguínea.

- Libera endorfina y serotonina.

- $\quad$ Proporciona la inclusión social.

Las actividades lúdicas pueden ser variadas, como: ejercicios físicos, mentales, destreza, equilibrio, entre otros.

En relación a los estudiantes, la práctica de un ejercicio lúdico es indispensable para su crecimiento personal y social, aparte de ser visto como una alternativa de ocio sana. Los beneficios que conlleva la realización de alguna actividad son: aumento de autoestima, desarrolla la creatividad y pensamiento, estimula la socialización, explora las posibilidades sensoriales y motoras, prepara al estudiante al mundo del trabajo, entre otras.

Por otro lado, el juego es visto como un método placentero para impartir educación. Los especialistas en el área de psicología y pedagogía, indican que el juego es una herramienta esencial para explotar y desarrollar todas las áreas de un individuo, y de ahí su importancia de realizar y seleccionar juegos adecuados al área que se desea instruir o desarrollar. Con respecto a este punto, el método lúdico puede ser empleado tanto para niños como adolescentes.

Karl Groos (1902) menciona que el juego es objeto de una investigación psicológica especial, siendo el primero en constatar el papel del juego como fenómeno de desarrollo del pensamiento y de la actividad. Por lo que la presente investigación se fundamenta en la teoría del juego como anticipación funcional. 


\section{Estrategia lúdica}

Según Melendres y Tocto (2010) la estrategia lúdica es una metodología de enseñanza de carácter participativo y dialógico impulsado por el uso creativo y pedagógicamente coherente, técnicas, ejercicios, historias y juegos educativos, creado específicamente para generar un aprendizaje significativo, tanto en términos de conocimientos, destrezas y habilidades sociales, como los valores de incorporación.

Tomando el juego desde el punto de vista educativo, implica que este se utiliza en muchos casos para manipular y control de los niños en el ámbito escolar en el que el aprendizaje a través de estas actividades como juegos o de juego; violando así la esencia y las características del juego como una experiencia cultural y como cámara de alumnos vinculados a experiencias de la vida. Desde este punto de vista el juego en el espacio libre al día es muy diferente al juego dentro de un regulado e institucionalizado como es el entorno escolar.

Este tipo de actividades, las estrategias son una dimensión del desarrollo humano que promueve el desarrollo psicosocial, la adquisición de conocimientos, la formación de la personalidad, y, es decir, que contienen una amplia gama de actividades donde el placer, el disfrute, la actividad creativa y el conocimiento obtienen juntos.

El grupo cree que las estrategias lúdicas son muy importantes para el desarrollo del pensamiento lógico de los niños y despertar su interés por aprender cosas nuevas y creativas para su vida diaria con la sociedad y por lo tanto ser capaz de hacer frente más adelante en sus estudios.

\section{Comprensión lectora}

Bugelski (1974) afirma que la lectura, "es el máximo logro del hombre, tal vez el invento más maravilloso de la mente humana y un proceso tan complejo que su comprensión equivaldría a entender cómo trabaja la mente" (390), pues tomando en cuenta la relación entre pensamiento y lenguaje, la lectura es fundamental para el lenguaje; mientras que Stern (1983) sostiene que la lectura de publicaciones científicas, motiva un justificado interés por el método de enseñanza de lenguas.

La construcción de un significado personal de la información que se encuentra en el texto mediante la puesta en acción de la decodificación de palabras, frases, párrafos, e ideas que el autor quiere transmitir, a través de la integración entre el texto y el lector. 
ISSN: 2600-5859

La Fundación BBVA (2011) sostiene que la comprensión de lectura es una habilidad básica sobre la cual una serie de capacidades relacionadas se desarrollan: la gestión de la vía oral, amor por la lectura, así como un pensamiento crítico. El desarrollo de habilidades para la comprensión de la lectura es una forma de que la provisión de herramientas para la vida académica, profesional y social de los estudiantes.

La comprensión de lectura es un indicador clave a la hora de hacer planes de clases para el desarrollo de las autoridades gubernamentales; como un indicador sensible de una educación de calidad. Una persona que entiende lo que es la lectura que va a poder lograr un mejor desarrollo profesional, técnico y social.

La falta de comprensión de lectura genera pobreza. Comprender lo que se lee es un requisito sustantivo para que un niño se convierta en un adulto progreso y contribuir al desarrollo de la familia y el país.

La comprensión de lectura es también un hecho de impacto en el negocio cuando se toman decisiones sobre los planes de inversión y desarrollo. De hecho, la capacidad que tiene el personal en diferentes jerarquías es un hecho de vital importancia para las empresas.

\section{La importancia de la lectura en inglés}

Pinza y Astudillo (2014) indican que muchos estudiantes están fascinados por el aprendizaje de un nuevo idioma y están entusiasmados con el estudio de inglés, pero pocos tienen éxito por diversas razones, entre ellas: es considerado un lenguaje universal y es necesario para un mejor futuro profesional.

La lectura es uno de los principales medios para el aprendizaje de una lengua, aunque otros recursos de apoyo como videos y tarjetas de memoria flash, la lectura es la forma más económica para acceder a un idioma extranjero que nos da un beneficio adicional, lo que permite al lector una la forma objetiva y eficaz ampliar su vocabulario.

La lectura en inglés tiene una gran importancia social, educativa y práctica, es una de las habilidades más trascendentes, pero a menudo la falta de motivación, estrategias y técnicas, se vuelve aburrido para el alumno y el profesor.

Mediante la lectura se puede saber acerca de la actividad económica, científica y cultural de un país, mientras que el vocabulario para entender que el lenguaje de una manera práctica aumenta. 
ISSN: 2600-5859

La lectura en inglés debe ser divertida como en español, pero hay muchos obstáculos en el momento de la comprensión de la lectura, por ejemplo, el vocabulario se puede presentar en diferentes niveles de lectura, y si el lector no está al mismo nivel, que puede llegar a ser muy complejas que causan dificultades de comprensión.

La tarea de la enseñanza requiere que los profesores tengan una formación adecuada y con formación pedagógica, así, por lo que su trabajo y la interacción con los estudiantes pueden ser beneficiosos para ambas partes.

A menudo, la falta de motivación viene de la esfera de los alumnos familia y esto influye en el bajo rendimiento, por tanto, los padres deben promover, animar y felicitar a todos los logros y el progreso de sus hijos.

La comprensión de lectura es un proceso complejo que identifica las palabras y significados, esta es la diferencia entre la lectura y la comprensión. Comprensión de lectura en inglés lleva mayor peso en el contexto del ejercicio de razonamiento y tiene como objetivo desarrollar la capacidad de leer analíticamente; constituye uno de los objetivos básicos de los métodos de enseñanza. Ejercicios aplicados adecuadamente la comprensión de lectura miden la capacidad de reconocer el significado de una palabra o frase en el contexto de otras ideas; la capacidad de comprender e identificar los fundamentos de la lectura; la capacidad de identificar las relaciones entre las ideas y, finalmente, el análisis y síntesis de la información.

Los tipos comunes de lectura de la prueba son:

Lectura visual de las palabras: las palabras se usan cada vez más difícil hasta que el lector no puede leer o entender lo que se presenta. El nivel de dificultad se maneja con un mayor número de letras o sílabas, usando relaciones menos comunes o complejas palabras fonéticas - pomológica.

Lectura de "no hay palabras". El uso de listas de sílabas pronunciables sin querer ser leído en voz alta. El aumento de dificultad se logra mediante secuencias más largas.

Comprensión lectora para el lector de un texto o pasaje del mismo, que se pueden leer en silencio o en voz alta se presenta. Entonces preguntas para evaluar lo que se ha entendido surgir.

Fluidez en la lectura. La velocidad se evaluó a las palabras de nombres.

La precisión de la lectura. Se evalúa la capacidad de nombrar correctamente las palabras en una página. 
ISSN: 2600-5859

\section{Metodología}

La investigación fue de tipo aplicada, pues se buscó resolver un problema en beneficio de la sociedad. Además, la investigación fue de campo, ya que se llevó a cabo en contacto directo con los hechos, y actores de la investigación.

La investigación tuvo un diseño transversal; ya que los datos fueron obtenidos en un solo momento, y con los resultados obtenidos se describieron las variables y analizó el impacto en un momento dado.

La investigación considero una muestra intencional, integrada por 30 estudiantes de segundo nivel de la carrera de Agroindustria de la Facultad de Ciencias Pecuarias de la Escuela Superior Politécnica de Chimborazo, durante el periodo académico marzo - agosto 2019. Los estudiantes son hombres y mujeres que tienen iguales conocimientos de inglés. Al ser la muestra pequeña e intencional representan la totalidad de la población.

La técnica fue la observación y el instrumento una ficha de observación, la misma que fue utilizada por el docente para registrar la opción por cada actividad lúdica que realiza cada estudiante.

Para la obtención de los datos se consideró las siguientes actividades lúdicas: Cuento, juego y Vocabulario. Por cada actividad lúdica, se incluyeron los siguientes indicadores donde cada uno de ellos, fueron evaluados mediante una escala de Linkert con las opciones de $\mathrm{Si}$, A veces y No.

Actividad lúdica: Cuento

- Comprensión de textos escritos en inglés de forma global y específica

- Interés de la actividad de la lectura de textos en inglés

- Anticipo al propósito del texto de inglés

- Respuesta a preguntas que se le plantean después de haber leído el texto de inglés

- Reconocimiento de características estructurales y gramaticales de un párrafo de inglés

- Escritura de resúmenes, acerca del texto de inglés con propia síntesis

Actividad lúdica: Juegos

- Discernimiento y selección de datos necesarios para responder una pregunta de inglés correctamente.

- Uso o asociación de dibujos para predecir el contenido de un texto de inglés

- Procesamiento de orden de lectura de inglés

- Escritura lógica gramatical en un texto de inglés 
- Resolución o creación de una solución en inglés a un problema planteado Actividad lúdica: Vocabulario

- Uso de conocimientos o información previa a un tema de inglés

- Exploración de las palabras desconocidas de inglés, en el diccionario

- Uso de nuevas palabras para formar una oración en inglés.

La ficha de observación fue elaborada, revisada y aprobada por especialistas del área. Quienes, en base a los indicadores elaboraron preguntas a fin de cuantificar el nivel alcanzado por cada actividad lúdica.

La intervención fue mediante un conjunto de actividades que fueron desarrolladas en base a un cronograma por parte del docente con los estudiantes en un periodo de 9 semanas. Las actividades fueron combinadas y al mismo tiempo evaluadas, para luego registrar los resultados, tabularlos, analizarlos e interpretados para finalmente realizar las respectivas conclusiones del alcance obtenido por la investigación.

El análisis de la información que permitió la interpretación de los datos, fue concebido de la siguiente manera:

- El docente mediante el plan de intervención desarrollo en las 9 semanas las actividades lúdicas donde cada estudiante fue observado y mediante la ficha de observación se registró la opción que se consideró pertinente.

- Los datos de las fichas de observación por cada estudiante, fue organizada e ingresada en tablas de una hoja de cálculo de Microsoft Excel.

- Los datos fueron tabulados y se estableció las frecuencias alcanzadas por cada opción, clasificadas según los indicadores por cada actividad lúdica, para posterior representarla en gráficos estadísticos.

- De los datos procesados, se procedió al análisis e interpretación general y por cada actividad lúdica.

Finalmente se establecieron las conclusiones obtenidas en la investigación 
ISSN: 2600-5859

\section{Resultados}

Tabla 1. Actividades lúdicas desarrolladas por los estudiantes

\begin{tabular}{|c|c|c|c|c|c|c|}
\hline \multirow{2}{*}{$\begin{array}{c}\text { ACTIVIDADES } \\
\text { LÚDICAS }\end{array}$} & \multirow[t]{2}{*}{ \# } & \multirow[t]{2}{*}{ INDICADORES } & \multicolumn{3}{|c|}{ SUBINDICADORES } & \multirow[t]{2}{*}{ TOTAL } \\
\hline & & & SI & A VECES & NO & \\
\hline \multirow[t]{6}{*}{ CUENTOS } & 1 & $\begin{array}{l}\text { ¿Comprende los textos escritos en inglés de } \\
\text { forma global y específica? }\end{array}$ & 19 & 6 & 5 & 30 \\
\hline & 2 & $\begin{array}{l}\text { ¿Se muestra interesado en la actividad de la } \\
\text { lectura de los textos en inglés? }\end{array}$ & 21 & 5 & 4 & 30 \\
\hline & 3 & ¿Se anticipa al propósito del texto de inglés? & 18 & 7 & 5 & 30 \\
\hline & 4 & $\begin{array}{l}\text { ¿Responde correctamente a las preguntas } \\
\text { que se le plantean después de haber leído el } \\
\text { texto de inglés? }\end{array}$ & 17 & 8 & 5 & 30 \\
\hline & 5 & $\begin{array}{l}\text { ¿Reconoce las características estructurales y } \\
\text { gramaticales de un párrafo de inglés? }\end{array}$ & 19 & 7 & 4 & 30 \\
\hline & 6 & $\begin{array}{l}\text { ¿Escribe resúmenes acerca del texto de } \\
\text { inglés con sus propias palabras? }\end{array}$ & 16 & 8 & 6 & 30 \\
\hline \multirow[t]{5}{*}{ JUEGOS } & 7 & $\begin{array}{l}\text { ¿Discierne y selecciona los datos necesarios } \\
\text { para responder una pregunta de inglés } \\
\text { correctamente? }\end{array}$ & 17 & 7 & 6 & 30 \\
\hline & 8 & $\begin{array}{l}\text { ¿Usa o asocia los dibujos para predecir el } \\
\text { contenido de un texto de inglés? }\end{array}$ & 20 & 7 & 3 & 30 \\
\hline & 9 & ¿Procesan un orden de lectura de inglés? & 18 & 7 & 5 & 30 \\
\hline & 10 & $\begin{array}{l}\text { ¿Escribe con lógica gramatical en un texto } \\
\text { de inglés? }\end{array}$ & 17 & 7 & 6 & 30 \\
\hline & 11 & $\begin{array}{l}\text { ¿Puede resolver e inventar una solución en } \\
\text { inglés a un problema planteado? }\end{array}$ & 16 & 7 & 7 & 30 \\
\hline \multirow[t]{3}{*}{ VOCABULARIO } & 12 & $\begin{array}{l}\text { ¿Usa los conocimientos o información } \\
\text { previa a un tema de inglés? }\end{array}$ & 16 & 6 & 8 & 30 \\
\hline & 13 & $\begin{array}{l}\text { ¿Busca las palabras desconocidas de inglés, } \\
\text { en el diccionario? }\end{array}$ & 20 & 6 & 4 & 30 \\
\hline & 14 & $\begin{array}{l}\text { ¿Usa nuevas palabras para formar una } \\
\text { oración en inglés? }\end{array}$ & 17 & 7 & 6 & 30 \\
\hline \multicolumn{3}{|l|}{ Promedio } & 18 & 7 & 5 & \\
\hline
\end{tabular}

Fuente: Test

Elaborado por: Pazmiño Luis 


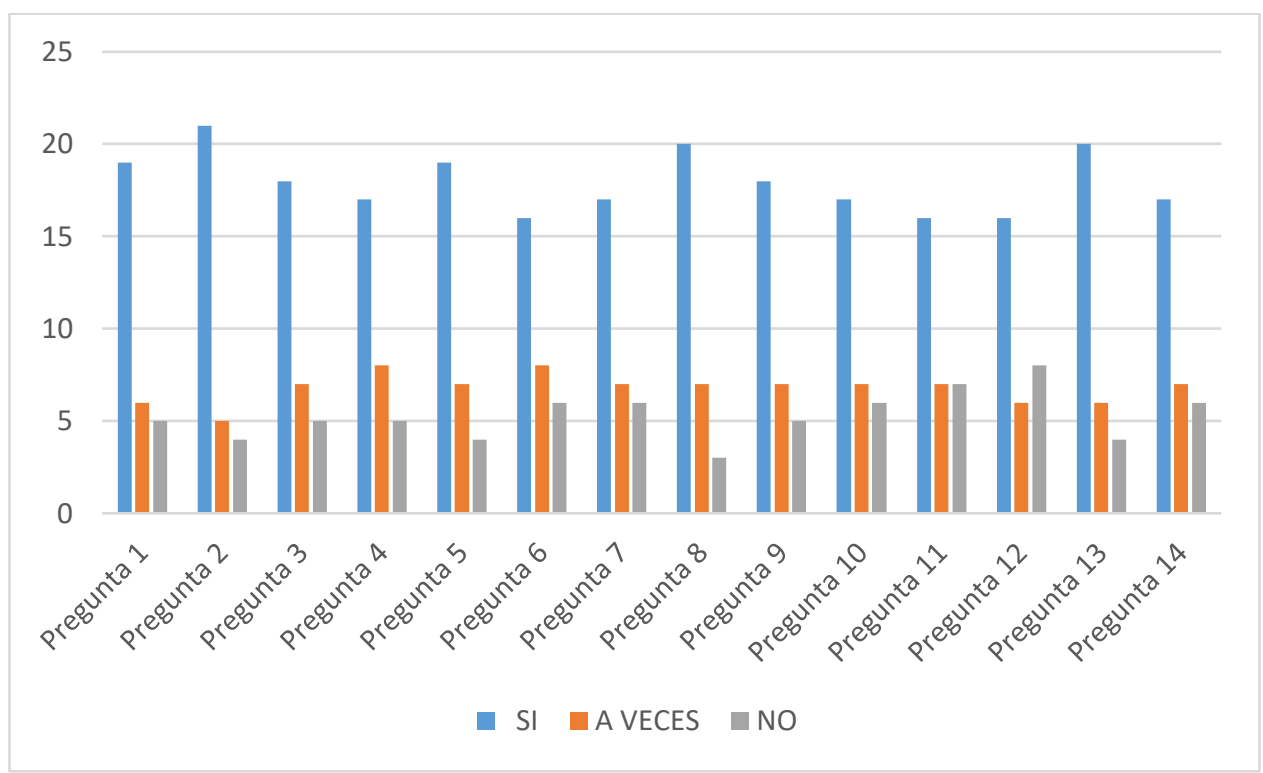

Figura 1. Actividades lúdicas desarrolladas por los estudiantes

\section{Análisis e interpretación}

Haciendo referencias a las frecuencias registradas de las actividades lúdicas, la opción SI a lo largo de las preguntas que representan los indicadores es tendencia con un promedio de 18 frecuencias, donde el puntaje mayor fue de 21 en la pregunta referente al interés en la actividad de la lectura de los textos en inglés en la actividad de los cuentos; mientras que la menor frecuencia se registró en tres indicadores donde se evaluó si la escritura de los resúmenes acerca del texto de inglés lo hacen a través de sus propias palabras; si puede el estudiante resolver e inventar una solución en inglés a un problema planteado; y, finalmente si el estudiante usa los conocimientos o información previa a un tema de inglés.

La opción A veces presentó un promedio de frecuencias de 7, valor que estuvo por debajo del $50 \%$ del promedio de la opción SI. La mayor frecuencia se presentó en los indicadores referentes a la que si los estudiantes, Responden correctamente a las preguntas que se le plantean después de haber leído el texto de inglés; si Escriben los resúmenes acerca del texto de inglés con sus propias palabras; mientras que la frecuencia más baja se presentó en el indicador que evaluó si los estudiantes muestran interés en la actividad de la lectura de los textos en inglés.

El No con respecto a las otras opciones, fue la opción que registró la menor frecuencia promedio con un valor de 5 puntos. La mayor frecuencia fue registrada en la actividad lúdica en el indicador, que evaluó si el estudiante usa los conocimientos o información previa a un tema de inglés; 
ISSN: 2600-5859

mientras que la menor frecuencia fue con respecto al indicador que evaluó si el estudiante usa o asocia los dibujos para predecir el contenido de un texto de inglés.

\section{Actividad lúdica: Cuentos}

Indicador 1. Del 100\% de los estudiantes evaluados, se constató que el 63\% de los estudiantes comprende los textos escritos en inglés de forma global y específica con la ayuda de los cuentos, el 20\% a veces; mientras que el $17 \%$ No entendió.

Indicador 2. Por otra parte, del 100\% de estudiantes, $70 \%$, es decir la mayoría se muestra interesado en la actividad de la lectura de los textos en inglés gracias al desarrollo de la actividad mediante los cuentos, $17 \%$ a veces; mientras que el $13 \%$ No.

Indicador 3. Con respecto al indicador 3, acerca de que, si el estudiante se anticipa al propósito del texto de inglés mediante cuentos, la mayoría con un porcentaje del $60 \%$ se encasilla en la opción SI, el 23\% veces; mientras que el 17\% NO.

Indicador 4. Al momento de responder correctamente a las preguntas que se le plantean después de haber leído el texto de inglés mediante los cuentos, del $100 \%$ de los estudiantes, $56 \%$ se ubican en la opción SI, el 27\% A veces; mientras que el 13\% en la opción NO. Es decir, esta opción fue la que registro la menor frecuencia la opción SI; por lo que se debe revisar y utilizar otras estrategias para fortalecer el indicador en mención.

Indicador 5. Del 100\% de los estudiantes, el 64\%, es decir la mayoría, reconocen las características estructurales y gramaticales de un párrafo de inglés mediante la actividad de los cuentos, el 23\% A veces; mientras que el $13 \%$ No reconoce.

Indicador 6. Del 100\% de los estudiantes, el 53\%, escriben resúmenes acerca del texto de inglés con sus propias palabras con la ayuda de los cuentos, el $27 \%$ a veces; mientras que el $20 \%$ No. Por lo que se debe fortalecer mediante alguna estrategia alternativa el indicador en mención para incidir y mejorar la escritura de los resúmenes.

La actividad lúdica de los cuentos, en comparación a las demás actividades en el contexto de la presente investigación, fue la que registro el mayor porcentaje de las frecuencias en los indicadores que se utilizaron para cuantificar la actividad.

\section{Actividad lúdica: Juegos}

Indicador 7. Al aplicar la actividad lúdica de los juegos, del 100\% de los estudiantes, el 57\% SI discierne y selecciona los datos necesarios para responder una pregunta de inglés correctamente, 
ISSN: 2600-5859

23\% A veces; mientras que el 20\% No discierne ni responde correctamente. Al igual que el indicador 6, se requiere fortalecer la actividad o alternar con otra estrategia.

Indicador 8. Del 100\% de los estudiantes, la mayoría con un el 67\%, SI usa o asocia los dibujos para predecir el contenido de un texto de inglés, el 23\% A veces, el 10\% registra la opción NO.

Indicador 9. El 60\% de los estudiantes, procesan de forma ordenada una lectura de inglés, el 23\% A veces; mientras que 17 NO. Es decir, existe un porcentaje aceptable en la forma ordenada de la lectura, sin embargo, requiere ser fortalecida.

Indicador 10. En cuanto a la escritura con lógica gramatical en un texto de inglés mediante la actividad lúdica del juego, del 100\% de los estudiantes, el 57\% registra la opción SI, el 23\% A veces, mientras que el $20 \%$ la opción NO.

Indicador 11. Del 100\% de los estudiantes evaluados en la actividad lúdica del juego, la mayoría con el 54\% puede resolver e inventar una solución en inglés a un problema planteado, el 23\% A veces; mientras que el $23 \%$ No.

Es recomendable que las actividades lúdicas del juego sean revisadas y actualizadas, pues la tabulación de los resultados con los estudiantes, implican frecuencias no significativas con respecto a la tendencia de la mayoría. Por lo que, debe ser mejoradas mediante alternativas que se orienten a mejorar las frecuencias de los indicadores que se evalúan para medir la actividad.

\section{Actividad lúdica: Vocabulario}

Indicador 12. Del 100\% de los estudiantes de la actividad lúdica del vocabulario, 53\% usa los conocimientos o información previa a un tema de inglés, el 27\% NO, mientras que el $20 \%$ registra A veces, Es decir fue el indicador que obtuvo mayores frecuencias en la opción NO, siendo importante tomar alternativas en la actividad para modificar el resultado obtenido.

Indicador 13. En cuanto al indicador donde se evalúa la actividad lúdica del vocabulario, el estudiante SI busca las palabras desconocidas de inglés en el diccionario con un 67\% de frecuencias, el 20\% A veces; mientras que el 13\% la opción NO. Sin duda la mayoría de los estudiantes hacen uso del diccionario.

Indicador 14. Del 100\% de los estudiantes, el 57\% SI usa nuevas palabras para formar una oración en inglés mediante la actividad de vocabulario, el $23 \%$ A veces; mientras que $20 \%$ no utiliza.

\section{Conclusiones}


ISSN: 2600-5859

- La intervención mediante las actividades lúdicas, al mismo tiempo que permitió incidir en la comprensión lectora del idioma inglés, también permitió conocer que el uso de las actividades lúdicas de la categoría de los Juego y vocabulario con respecto a los cuentos, obtuvieron resultados que deben ser fortalecidos o reemplazados con otro tipo de estrategias o alternativas para mejorar las frecuencias de la opción favorable. Por lo que es necesario realizar una nueva exploración a través de una investigación para contrastar con los resultados obtenidos con la actual investigación.

- Los cuentos, juegos interactivos y vocabulario como estrategias para la comprensión lectora del idioma inglés motivan y generan interés en los estudiantes de segundo nivel y se ha visto reflejado en su rendimiento académico y que inciden en su capacidad de síntesis y el aumento del léxico, es decir que los docentes deben tomar esta estrategia para incidir positivamente e incorporarlos en su planificación de actividades académicas.

- La opción SI, al momento de tabular las actividades lúdicas con relación a los indicadores, fue tendencia en las 14 preguntas que se plantearon para evaluar la comprensión lectora del idioma inglés; mientras que la opción No fue la opción que menos frecuencias registro, por lo que los indicadores fueron acertados para medir el alcance de la investigación.

\section{Referencias Bibliográficas}

Bugelski, BR. (1974). Psicología del Aprendizaje Aplicada a la Enseñanza. Madrid. Taller de Ediciones.

Díaz, F. \&. (2002). Estrategias docentes para un aprendizaje significativo: una interpretación constructivista. México: Mc Graw Hill.

Kintsch, W. (1998). Comprehension: A paradigm for cognition. Nueva York: Cambridge University Press.

Melendres, N., \& Tocto, A. (2010). Estrategias Lúdicas en el desarrollo del pensamiento lógico de los niños y niñas de tres a cuatro años del Centro de Desarrollo Infantil "Corazón de Jesús" de la Comunidad de Santa Rosa de Totoras del Cantón San Miguel, Provincia Bolívar, durante el año. Guaranda: Universidad Estatal de Bolívar

Groos, K. (1902). The Play of man. Appleton. N. York. 
ISSN: 2600-5859

Fundación BBVA. (2011). ¿Por qué es importante la comprensión lectora? http://www.leer.pe/ique-hacemos/ipor-quees-importante-la-comprension-lectora.

Pinza, A., \& Astudillo, A. (2014). La importancia de la lectura en el idioma inglés aplicando técnicas y lecturas motivadoras. Guayaquil - Ecuador: Universidad Tecnológica Empresarial de Guayaquil.

STERN, H.H. (1983): Fundamental Concepts of Language Teaching. Oxford. Oxford. U. Press.

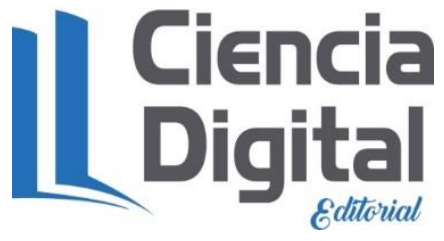




\section{PARA CITAR EL ARTÍCULO INDEXADO}

Pazmiño Pavón, L. E., Armas Pesántez, P. R., Salazar Calderón, E. H., \& Heredia Arboleda, E. E. (2020). La comprensión lectora del idioma inglés mediante actividades lúdicas. ConcienciaDigital, 3(1.1), 103-117. https://doi.org/10.33262/concienciadigital.v3i1.1.1134

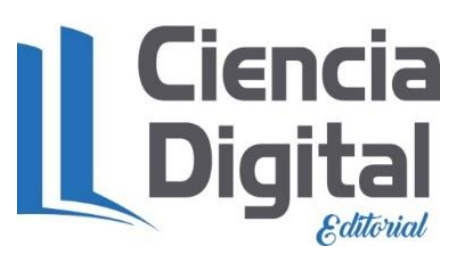

El artículo que se publica es de exclusiva responsabilidad de los autores y no necesariamente reflejan el pensamiento de la Revista Conciencia Digital.

El artículo queda en propiedad de la revista y, por tanto, su publicación parcial y/o total en otro medio tiene que ser autorizado por el director de la Revista Conciencia Digital.
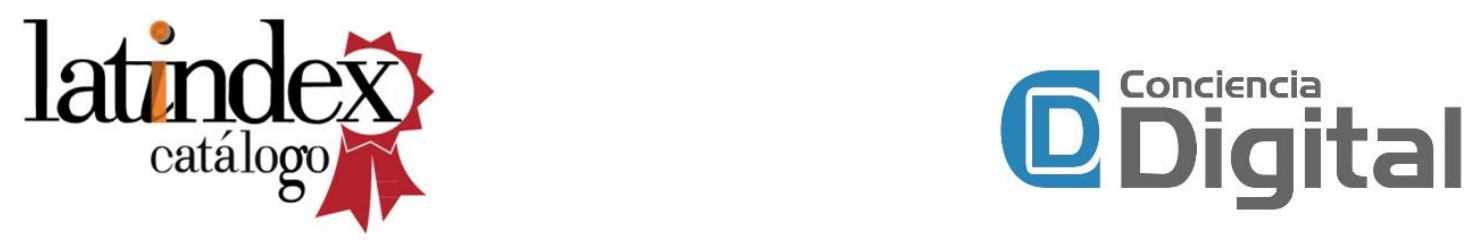